\title{
What potential is there for liposomal- based nanotherapy for the treatment of Type 1 diabetes?
}

\author{
"We believe that liposomal-based immunotherapy could reestablish \\ immunological tolerance and is a promising strategy to arrest \\ autoimmune destruction in Type 1 diabetes and other autoimmune \\ diseases."
}

Keywords: autoimmunity $\bullet$ immunotherapy $\bullet$ liposomes $\bullet$ nanomedicine $\bullet$ Type 1 diabetes

\section{Type 1 diabetes \& current immunotherapies}

Type 1 diabetes (T1D) is a chronic metabolic disease that results from the autoimmune attack against insulin-producing $\beta$-cells in the islets of Langerhans of the pancreas. The etiology of the disease is unknown and the patients require exogenous insulin administration, but the control of glycemia is not always easy to achieve. The long preclinical asymptomatic period and the difficult access to the target organ have impaired the understanding of the causes and the finding of a cure for the disease. Currently, there is no treatment to restore endogenous insulin secretion in patients with T1D and the incidence of the disease is increasing by $3-4 \%$ per year in developing countries, especially among young children. Therefore, there is a need for improved immunotherapies to arrest the autoimmune destruction and to induce long-term tolerance, avoiding systemic side effects and allowing $\beta$-cell regeneration.

During the last decades, many clinical trials using immunotherapies for T1D have been developed. Unfortunately, none of these approaches has been able to prevent or cure human T1D. Some innovative strategies to develop immunomodulatory agents for T1D are based on nanotechnology and use nanoparticles not only as delivery systems but also as immunomodulatory agents [1].

\section{Liposomal-based nanotherapy inspired by a physiological process}

It is well known that apoptotic cells are a source of autoantigens that maintain toler- ance to self-after their removal by phagocytes [2]. However, apoptotic cells contribute to T1D due to too much loss of $\beta$-cells or too slow apoptotic cell removal [3]. When dying $\beta$-cells are not rapidly removed, they turn into necrotic cells and release proinflammatory molecules that may activate antigen presenting cells (APCs), such as dendritic cells (DCs), contributing to autoimmunity. The suppressive function of DCs after the uptake of apoptotic cells constitutes a strategy to arrest autoimmunity [4]. The ability of apoptotic $\beta$-cells to arrest the autoimmune attack was demonstrated in the experimental model of T1D, the nonobese diabetic (NOD) mouse, which spontaneously develops the disease and has served as a model for understanding the immunological and genetic basis of T1D and to test immunotherapies [5].

The transfusion of apoptotic islet cells decreased diabetes incidence in NOD mice [6], and the administration of islet apoptotic cells loaded into tolerogenic DCs prevented T1D in the same experimental model [7]. These tolerogenic features of apoptosis have been the basis for a liposomal-based antigen-specific immunotherapy, developed to mimic apoptotic $\beta$-cells.

Liposomes are artificially constructed micro- or nanobubbles - usually between 20 and $1000 \mathrm{~nm}$ in diameter - with an aqueous core encapsulated by phospholipids. This structure provides liposomes with the ability to carry and protect encapsulated molecules; hydrophilic molecules are located in the core and hydrophobic ones are placed within the
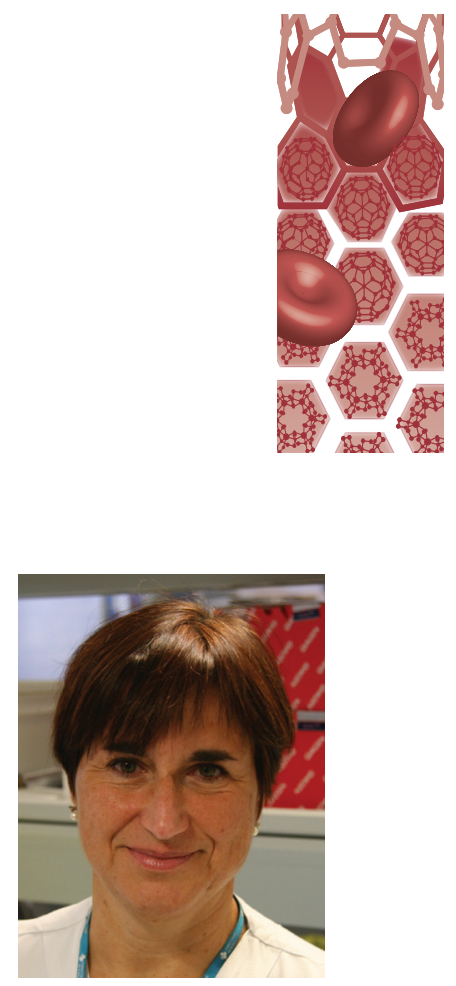

Marta Vives-Pi

Author for correspondence: Immunology Department, CIBER of Diabetes \& Associated Metabolic Diseases (CIBERDEM), Germans Trias i Pujol Research Institute, Universitat Autonoma de Barcelona, Badalona, Spain

Tel.: +34934978666

Fax: +34934978668 mvives@igtp.cat

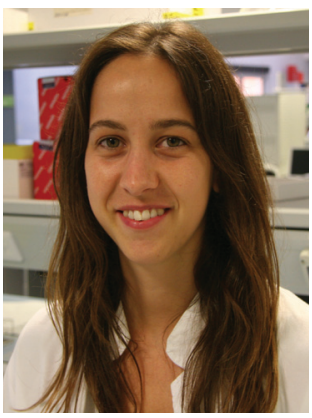

Irma Pujol-Autonell Immunology Department, CIBER of Diabetes \& Associated Metabolic Diseases (CIBERDEM), Germans Trias i Pujol Research Institute, Universitat Autonoma de Barcelona, Badalona, Spain

Future $\because$ Medicine part of 
lipid bilayers. Liposomes were first described in 1965 and proposed as drug delivery system. Since then, liposome formulations have progressed [8]. Liposomes have a great potential in the medical field due to their ability to cross cell membranes and have led to numerous clinical trials in cancer, infection, inflammation, immunosuppression and vaccines [9]. Some of these agents are the basis of current commercial products and many more in the pipeline. Hence, liposomes can be engineered specifically to avoid, inhibit or enhance the activity of the immune system. Particle size, surface charge, hydrophobicity/hydrophilicity and design - surface and encapsulated molecules - can determine the effects in the immune response and should be carefully chosen. Liposomes have the advantage that they may be customized for a specific purpose and may be combined with specific ligands, such as antibodies, peptides and glycoproteins, to target their deliver to APCs, thus modulating the immune response. In the case of T1D, liposomes have been designed to arrest the autoimmune attack and to restore tolerance to self.

\section{"Therapeutic applications of liposomes in autoimmune diseases have been reported for rheumatoid arthritis and multiple sclerosis, but none of these strategies include phosphatidylserine in the liposomal formulation."}

Phosphatidylserine (PS) is a molecule normally located on the inner leaflet of the cell membrane and is exposed in the outer leaflet of cells during apoptosis, constituting the main 'eat me' signal that triggers phagocytosis [10]. PS is recognized by innate receptors on the surface of phagocytes, and not only plays an important role in efferocytosis but also acts as a 'tolerate me' signal in APCs, promoting the release of anti-inflammatory molecules and inducing tolerogenic presentation of autoantigens [11,12]. Therefore, PS-liposomes can be used for immunomodulation, to reduce immunogenicity of encapsulated molecules. In fact, when FVIII - a protein that is deficient in hemophilia A - was encapsulated in PS-liposomes, its immunogenicity was reduced [13]. By contrast, PS is a molecule to avoid in the design of liposomal vaccines to stimulate the immune response against pathogens or tumors [14], due to its anti-inflammatory and tolerogenic effect.

Liposomes for T1D should be designed to mimic the tolerogenic effects of apoptotic cells after phagocytosis by the APCs, taking into account several necessary features [15]. First, particle size is essential to trigger phagocytosis and to avoid cell fusion. Large particles (>500 nm diameter) are phagocytosed more efficiently than smaller particles. Second, cationic or anionic particles are more attractive to phagocytes than neutral particles affecting also the immune system. Third, PS exposition on the outer liposome membrane is crucial for the discrimination between viable and apoptotic cells, the uptake of particles by phagocytes and the induction of 'tolerate me' signals during antigen presentation. Fourth, relevant T1D autoantigens should be encapsulated in liposomes in order to mimic apoptotic cells and to be presented in a tolerogenic manner in the context of the major histocompatibility complex (MHC) molecules. And finally, multivesicular liposome morphology has advantages regarding encapsulation and immunological activity.

\section{Future prospects for liposomal therapy in T1D}

Based on the immunomodulatory effects of apoptosis, we hypothesized that apoptotic mimicry can help to restore tolerance lost in T1D. To investigate this aspect, PS-containing liposomes were loaded with insulin peptides as autoantigens [16] to simulate apoptotic $\beta$-cell recognition by APCs. Liposomes were prepared in the size range for an efficient phagocytosis and with multivesicular vesicles morphology. The effect of antigenspecific liposomes in the reestablishment of peripheral tolerance was assessed in NOD mice. We have demonstrated that antigen-specific PS-liposomes induce tolerogenic DCs, promote prostaglandin $\mathrm{E}_{2}$ secretion - a tolerogenic mediator of APCs - and impair autoreactive $\mathrm{T}$ cell proliferation. When administered to prediabetic NOD mice, liposome signal was detected in the pancreas and draining lymph nodes. Importantly, this immunotherapy arrests the autoimmune aggression, reduces the severity of insulitis and prevents T1D by apoptotic mimicry [17]. Since antigen-specific PSliposomes resemble apoptotic cells in inhibiting maturation and immunostimulatory function of DCs, we believe that liposomes could be optimum vehicles to restore tolerance to autoantigens, thus constituting a promising strategy for autoimmune diseases.

We are fully aware that the NOD mouse strain has immunologic variability when compared with human. Moreover, the prevention of the disease in this model is relatively easy to achieve. Obviously, caution is needed when translating results from animal models to a clinical setting. First, diabetic patients do not share all the immune defects existing in the NOD mouse. Second, human $\beta$-cells are less able to regenerate than rodent $\beta$-cells. Third, NOD mice, unlike humans, are genetically identical. An important preclinical step will be the assessment of the in vitro effects of liposomes in human DCs from healthy subjects and T1D patients.

However, from our point of view, the added benefit of PS-liposome approach is the delivery of autoantigens along with PS, a combination to induce long-term spe- 
cific tolerance. Therapeutic applications of liposomes in autoimmune diseases have been reported for rheumatoid arthritis and multiple sclerosis [18], but none of these strategies include PS in the liposomal formulation. PS-liposomes can be nontoxic, biodegradable, biocompatible, easy to prepare and to customize, and low-cost. Moreover, PS-liposomes efficiently encapsulate and protect autoantigens and interact with APCs in a predictable manner, delivering the autoantigens in the cytosol and promoting MHC presentation.

Nevertheless, further research is needed to improve the effects of liposomal-based immunotherapy in T1D. Future efforts should be put into guiding translational research to successful clinical therapy. Although humans and NOD share many autoantigens, liposomes should be adapted for therapeutic purposes in human disease using human peptides. However, this immunotherapy could be improved by evaluating other candidate autoantigens for liposome encapsulation in terms of solubility, size and antigenicity. The role of liposomes in the reversal of the disease should also be investigated, alone or in combination with regenerative strategies. Moreover, routes of liposome delivery and conditions that could influence liposome biodistribution should be taken into consideration to promote their action in the islets and to minimize side effects.

The incoming data on the pathogenesis of the disease [19] will help to improve liposome design to opti-

\section{References}

Papers of special note have been highlighted as:

- of interest; $\bullet \bullet$ of considerable interest

1 Garciafigueroa Y, Trucco M, Giannoukakis N. A brief glimpse over the horizon for Type 1 diabetes nanotherapeutics. Clin. Immunol. pii: S1521-6616(15)00116-3. doi: 10.1016/j. clim.2015.03.016 (2015) (Epub ahead of print).

- Review on innovative approaches based on nanotherapeutics for Type 1 diabetes.

2 Nagata S, Hanayama R, Kawane K. Autoimmunity and the clearance of dead cells. Cell 140(5), 619-630 (2010).

-• Comprehensive discussion of the mechanisms of apoptotic cells to maintain homeostasis and to induce tolerance to self.

3 Vives-Pi M, Rodriguez-Fernandez S, Pujol-Autonell I. How apoptotic beta-cells direct immune response to tolerance or to autoimmune diabetes: a review. Apoptosis 20(3), 263-272 (2015).

4 Mbongue J, Nicholas D, Firek A, Langridge W. The role of dendritic cells in tissue-specific autoimmunity. J. Immunol. Res. 2014, 857143 (2014).

5 Reed JC, Herold KC. Thinking bedside at the bench: the nod mouse model of T1DM. Nat. Rev. Endocrinol. 11(5), 308-314 (2015).

6 Xia CQ, Peng R, Qiu Y, Annamalai M, Gordon D, ClareSalzler MJ. Transfusion of apoptotic beta-cells induces immune tolerance to beta-cell antigens and prevents Type 1 mize the therapeutic use of PS-liposomes. We believe that the optimization of this immunotherapy that has proven capable to re-establish peripheral immunological tolerance, in combination with anti-inflammatory agents and helping $\beta$-cell regeneration, will contribute to improve T1D treatment. This is an example of how this newly developed liposomal formulation can be utilized in T1D. We believe that liposomal-based immunotherapy could reestablish immunological tolerance and is a promising strategy to arrest autoimmune destruction in T1D and other autoimmune diseases.

\section{Acknowledgements}

The authors thank Daniel Maspoch and Mary Cano-Sarabia, from the Catalan Institute of Nanotechnology and Nanoscience (ICN2), for fruitful discussions. Special thanks to Deborah Cullell-Young for English grammar assistance.

\section{Financial \& competing interests disclosure}

CIBER of Diabetes and Associated Metabolic Diseases (CIBERDEM) is an initiative from Instituto de Salud Carlos III. The authors have no other relevant affiliations or financial involvement with any organization or entity with a financial interest in or financial conflict with the subject matter or materials discussed in the manuscript apart from those disclosed.

No writing assistance was utilized in the production of this manuscript.

diabetes in nod mice. Diabetes 56(8), 2116-2123 (2007).

7 Marin-Gallen S, Clemente-Casares X, Planas R et al. Dendritic cells pulsed with antigen-specific apoptotic bodies prevent experimental Type 1 diabetes. Clin. Exp. Immunol. 160(2), 207-214 (2010).

8 Chang HI, Yeh MK. Clinical development of liposomebased drugs: formulation, characterization, and therapeutic efficacy. Int. J. Nanomedicine 7, 49-60 (2012).

- Summary of the preclinical and clinical data related to liposomal formulations and their physicochemical properties.

9 Allen TM, Cullis PR. Liposomal drug delivery systems: from concept to clinical applications. Adv. Drug Deliv. Rev. 65(1), 36-48 (2013).

10 Hochreiter-Hufford A, Ravichandran KS. Clearing the dead: apoptotic cell sensing, recognition, engulfment, and digestion. Cold Spring Harbor Perspect. Biol. 5(1), a008748 (2013).

11 Wu Z, Nakanishi H. Phosphatidylserine-containing liposomes: potential pharmacological interventions against inflammatory and immune diseases through the production of prostaglandin E(2) after uptake by myeloid derived phagocytes. Arch. Immunologiae Therapiae Experimentalis 59(3), 195-201 (2011).

12 Zhong K, Song W, Wang Q et al. Murine myeloid dendritic cells that phagocytose apoptotic $\mathrm{T}$ cells inhibit the immune response via no. PLoS ONE 7(11), e 49378 (2012). 
13 Ramani K, Miclea RD, Purohit VS, Mager DE, Straubinger RM, Balu-Iyer SV. Phosphatidylserine containing liposomes reduce immunogenicity of recombinant human factor VIII (RFVIII) in a murine model of hemophilia A. J. Pharmaceut. Sci. 97(4), 1386-1398 (2008).

14 Watson DS, Endsley AN, Huang L. Design considerations for liposomal vaccines: influence of formulation parameters on antibody and cell-mediated immune responses to liposome associated antigens. Vaccine 30(13), 2256-2272 (2012).

15 Zolnik BS, Gonzalez-Fernandez A, Sadrieh N, Dobrovolskaia MA. Nanoparticles and the immune system. Endocrinology 151(2), 458-465 (2010).

16 Arvan P, Pietropaolo M, Ostrov D, Rhodes CJ. Islet autoantigens: structure, function, localization, and regulation. Cold Spring Harb. Perspect. Med. 2(8), a007658 (2012).
17 Pujol-Autonell I, Serracant-Prat A, Cano-Sarabia M et al. Use of autoantigen-loaded phosphatidylserine-liposomes to arrest autoimmunity in Type 1 diabetes. PLoS ONE 10(6), e0127057 (2015).

- Describes the use of liposomes rich on phosphatidylserine displaying insulin peptides to arrest autoimmunity in Type 1 diabetes. This is the first report on Type 1 diabetes prevention using liposomes.

18 Gharagozloo M, Majewski S, Foldvari M. Therapeutic applications of nanomedicine in autoimmune diseases: from immunosuppression to tolerance induction. Nanomedicine 11(4), 1003-1018 (2015).

19 Vehik K, Ajami NJ, Hadley D, Petrosino JF, Burkhardt BR. The changing landscape of Type 1 diabetes: recent developments and future frontiers. Curr. Diab. Rep. 13(5), 642-650 (2013). 